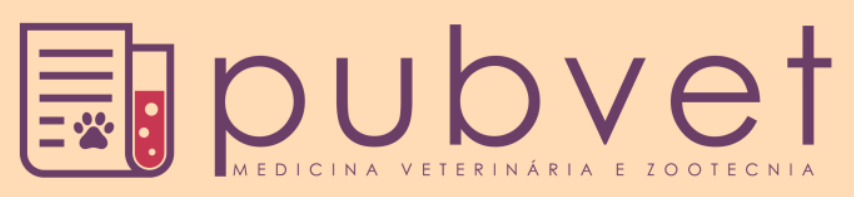

HTTP://DX.DOI.ORG/10.22256/PUBVET.V11N1.18-29

\title{
Bem-estar de ovinos: Revisão
}

\author{
Ana Carolina Barros de Freitas ${ }^{1 *}$, Celia Raquel Quirino², Rosemary Bastos ${ }^{2}$ \\ ${ }^{I}$ Doutoranda em Ciência animal na Universidade Estadual do Norte Fluminense Darcy Ribeiro/UENF, Laboratório de \\ Reprodução e Melhoramento Genético Animal. Campos dos Goytacazes - RJ, Brasil.e-mail: aninhabdf@hotmail.com \\ ${ }^{2}$ Docentes da Universidade Estadual do Norte Fluminense Darcy Ribeiro/UENF, Laboratório de Reprodução e Melhoramento \\ Genético Animal.Campos dos Goytacazes - RJ, Brasil.e-mail: crqster@gmail.com; rosebast@gmail.com \\ *Autor para correspondência
}

RESUMO. O bem-estar dos animais de produção é um tema recorrente. A facilidade de acesso às informações acerca dos métodos de produção das espécies criadas para fornecer alimento aos humanos faz com que a preocupação com os animais seja relevante para a aceitação de tais produtos pelos consumidores e sua consequente aquisição. Além da crescente exigência dos consumidores, é sabido que animais com bom estado de bem-estar apresentam melhor saúde, produtividade e fornecem produtos com qualidade superior. Com isso, é evidente que os produtores devem satisfazer as necessidades dos animais, tornando os produtos aceitáveis pelos consumidores e aumentando a lucratividade do sistema. Apesar dos ovinos terem sido uma das primeiras espécies domesticadas pelo homem, no Brasil ainda não estão disponíveis recomendações ou legislação específica que visem melhorar o bem-estar desta espécie. Esta revisão de literatura tem o objetivo de expor os principais conceitos e as recomendações para o bem-estar de ovinos. Estas recomendações estão fundamentadas em diversas pesquisas científicas voltadas para o bem-estar de ovinos e fornecem subsídios para o desenvolvimento de técnicas de manejos que visem melhorar a qualidade de vida dos animais.

Palavras chave: Conceitos, legislação, ovinocultura, recomendações

\section{The welfare of sheep: Review}

ABSTRACT. The welfare of livestock production is a recurring theme. Ease of access to information on the production methods of species farmed to provide food to human make concern about animals is relevant to the acceptance of such products by consumers and its subsequent acquisition. In addition to the growing demands of consumers, it is known that animals with good welfare state have better health, productivity and provide high quality products. By these means, it is clear that producers must satisfy the animals' needs, making the products acceptable to consumers and increasing the system's profitability. In spite of the sheep have been one of the first species domesticated by humans, in Brazil are still not available recommendations or specific legislation for improving the welfare of this species. This literature review aims to expose the main concepts and recommendations for the welfare of sheep. These recommendations are based on various scientific researches for the welfare of sheep and provide subsidies for the development of handling techniques to improve the quality of life of the animals.

Keywords: concepts, legislation, sheep breeding, recommendations

\section{El bienestar de las ovejas: Revisión}

RESUMEN. El bienestar de los animales de producción es un tema recurrente. La facilidad de acceso a las informaciones sobre los métodos de producción de las especies criadas para 
proporcionar alimento a los humanos hace con que la preocupación con os animales sea pertinente para la aceptación de tales productos por los consumidores y la consecuente adquisición. Además del aumento de la exigencia de los consumidores, es sabido que animales con buen estado de bienestar presentan mejores condiciones de salud, productividad y ofrecen productos con calidad superior. Así, es obvio que los productores deben satisfacer las necesidades de los animales, tornando los productos aceptables por los consumidores y aumentando la rentabilidad del sistema. A pesar de los ovinos haber sido una de las primeras especies domesticadas por el hombre, en Brasil aún no hay disponibilidad de recomendaciones o legislación específica que visen mejorar el bienestar de ésta especie. El objetivo de esta revisión de literatura es exponer los principales conceptos y recomendaciones para el bienestar de ovinos. Estas recomendaciones están fundamentadas en diversas investigaciones científicas enfocadas para el bienestar de ovinos y proporciona subsidios para el desarrollo de técnicas de manejos que visen mejorar la calidad de vida de los animales.

Palabras clave: Conceptos, legislación, ganadería ovina, recomendaciones

\section{Introdução}

A globalização, como um fenômeno ou processo mundial de integração ou partilha de informações, vem aumentando a disponibilidade e a velocidade de acesso às informações. Com isso, inúmeras pessoas podem acessar os mais diversos tipos de conteúdos a partir da internet, onde e quando quiserem. Esse fenômeno tem proporcionado mudanças na percepção da população sobre a qualidade do alimento ingerido e, como consequência, está aumentando a exigência por produtos "limpos, verdes e éticos" Pinheiro (Pinheiro \& Brito, 2009). A ovinocultura é uma alternativa interessante para os diferentes ecossistemas brasileiros, desde que seja explorada racionalmente e em sintonia com aspectos agroecológicos, econômicos e sociais (Simplício et al., 2004).

Os ovinos podem ser utilizados para diversos fins, como para a produção de lã, carne, leite e pele. Mas devem ter suas necessidades supridas para que produzam de forma eficiente, proporcionem lucro para seus produtores, tenham uma boa qualidade de vida e se transformem em produtos de qualidade para os consumidores. As boas condições de bem-estar dos animais exigem que sejam prevenidas ou tratadas as doenças; que haja proteção, manejo e alimentação corretos, além de serem abatidos ou eutanasiados de forma humana (OIE, 2014).

O Brasil, no papel de grande produtor e exportador de proteína de origem animal, deve ficar atento às exigências do mercado ou poderá sofrer prejuízos marcantes na sua economia (Pinheiro and Brito, 2009).

É de fundamental importância que sejam ministradas disciplinas de bem-estar animal e etologia nos cursos de Medicina Veterinária e Zootecnia para garantir que os profissionais sejam capazes de lidar com os animais da melhor forma possível e para que possam compartilhar e disseminar os conhecimentos adquiridos com os produtores e tratadores.

\section{Bem-estar animal (BEA)}

A preocupação atual com o bem-estar animal provém do fato de que a busca pelo aumento da produção de alimento e a necessidade da diminuição do custo de produção acarretaram no desenvolvimento de tecnologias cada vez mais eticamente inaceitáveis nos sistemas de produção (Pinheiro and Brito, 2009). Embora medidas para melhorar o bem-estar e a produtividade estejam facilmente disponíveis (Waterhouse, 1996) avanços ocorrem em maior velocidade quando esses benefícios estão combinados com preocupações públicas, vantagens econômicas e quando existem redes para facilitar a transferência de informações e coordenar atividades em setores comerciais e legislativos (Millman et al., 2004).

O preço não é mais o único fator avaliado pelos consumidores durante a escolha de um produto. Eles têm exigido uma conduta humanitária no tratamento dos animais em todas as etapas produtivas (na propriedade, durante o transporte $\mathrm{e}$ o no abate) e as exigências dos consumidores devem ser atendidas.

Antigamente produtos rotulados como "de qualidade" apresentavam aparência visual, vida de prateleira e sabor adequados (Waterhouse, 1996). Atualmente a "qualidade" depende da saúde animal (componente chave do bem-estar animal e fator crítico para atingir acesso ao mercado), das condições de higidez, da ausência de resíduos 
(medicamentos e hormônios), e das condições de bem-estar animal (Pinheiro and Brito, 2009). Sendo assim, os sistemas de produção percebidos como amigáveis aos animais (que proporcionam bom estado de bem-estar durante a vida) devem ser mais fáceis de manter do que sistemas menos amigáveis (Waterhouse, 1996).

Considerações éticas não excluem oportunidades de negócios e a busca por padrões mais elevados de bem-estar animal pode ser uma oportunidade de negócio que não deve ser desperdiçada devido a uma chance de penetrar em um mercado em constante crescimento (Pinheiro and Brito, 2009). Hoje existem dois grandes grupos de produtores rurais no Brasil: aqueles que conseguirão se adaptar e manterão a competitividade neste enorme mercado e os que serão marginalizados no processo por não seguirem as exigências da natureza e do consumidor (Euclides Filho, 2004).

O bem-estar é uma questão multicultural e multidimensional e é muito importante incluí-lo como tema central na educação veterinária e promover a investigação com base científica para normas e legislação de bem-estar animal (OIE, 2014). Estudos têm demonstrado que o estresse excessivo pode levar à baixa produção, afetar o crescimento ou a reprodução e aumentar a incidência de doenças, além da produção de carne com qualidade inferior (Pinheiro and Brito, 2009). Abordagens para minimizar os riscos de bem-estar devem levar em consideração algumas variáveis, tais como: comportamento maternal, comportamento de pastejo, resistência a doenças, reatividade comportamental, longevidade, sucesso reprodutivo, mortalidade, desafios nutricionais e térmicos, dor, liberdade comportamental, genética e ambiente (Lawrence and Appleby, 1996, Waterhouse, 1996, Wolf et al., 2008). Com essas medidas, será mais fácil defender práticas aceitáveis e argumentar para a modificação ou eliminação de sistemas inaceitáveis, independentemente do grau de consciência por parte dos consumidores sobre os problemas do bem-estar animal (Waterhouse, 1996).

\section{Tratadores}

Os tratadores são peça fundamental na produção animal, devendo saber o que estão fazendo e ser competentes para exercer suas responsabilidades. Cabe ao produtor rural fornecer segurança, infraestrutura e informações necessárias para o trabalho adequado e eficiente de seus funcionários (Pinheiro and Brito, 2009).

Segundo as diretrizes do Code of Recommendations for the Welfare of Livestock: Sheep (2013), os tratadores devem:

1. Estar cientes das necessidades de bemestar e ser capazes de assegurá-la a todos os animais do rebanho;

2. Executar uma rotina de cuidados após treinamento (por pessoas mais experientes ou em cursos);

3. Ter tempo suficiente para inspecionar todos os animais e intervir caso seja necessário; 4. Conhecer os sinais de boa saúde (estado de alerta geral, movimentação, alimentação e ruminação ativas, ausência de claudicação, ferimentos visíveis ou abscessos).

5. Conhecer os sinais de doenças (apatia, postura e comportamento anormais, claudicação, diarreia, ausência de ruminação, tosse persistente ou respiração ofegante, coçar e esfregar frequentemente, rápida perda de condição corporal, perda excessiva de lã, queda súbita na produção de leite e, em algumas circunstâncias, ficar afastado do rebanho).

6. Ter ajuda extra em épocas de maior trabalho (parto, tosquia) ou quando os funcionários regulares não puderem estar presentes (férias ou doença).

\section{Interação homem vs. animal}

Em um sistema de produção de ovinos a relação homem vs. animal deve ser otimizada, pois interações negativas podem diminuir o grau de bem-estar, prejudicar a saúde de ambas as espécies e podem trazer consequências indesejáveis à produtividade animal e à satisfação dos humanos com relação ao ambiente de trabalho. A relação entre humanos e animais está intimamente associada a vários aspectos de bem-estar animal (Probst et al., 2012), pois pessoas interagem com animais em todos os sistemas de produção, podendo ser um importante fator estressante dependendo do tipo de manipulação que executa (Romeyer and Bouissou, 1992). A natureza e as consequências de tais interações podem explicar os efeitos de alguns fatores estressantes do manejo sobre os processos reprodutivos, metabólicos e imunológicos dos animais (Carenzi and Verga, 2009).

Apesar de viverem próximos à humanos, a forma como os animais domésticos os percebem permanece pouco conhecida, mas é sabido que 
eles podem, eventualmente, integrar os humanos em sua rede social (Rault et al., 2011). Por conseguinte, a relação entre homens e animais pode ter importante efeito no comportamento, produtividade e bem-estar de animais de produção (Carenzi and Verga, 2009, Krohn et al., 2001).

A relação homem $v s$. animal é um equilíbrio entre interações positivas e negativas e, obviamente, os ovinos não receberão apenas contatos positivos, também experimentarão manipulações negativas, tais como castração, vacinação, caudectomia e tosquia durante a vida (Boivin et al., 2001). Com o fortalecimento do bem-estar, métodos de manejo não invasivos e que não deixam efeitos comportamentais negativos no rebanho têm sido buscados e o desenvolvimento de técnicas que aumentem a produção em combinação com o fornecimento de um grau satisfatório de bem-estar dos animais é possível através dos resultados provenientes de pesquisas (Pinheiro and Brito, 2009). Todavia, a dificuldade de interpretação é a maior limitação das pesquisas sobre bem-estar (Barnett and Hemsworth, 1990).

Carenzi and Verga (2009) explicam que os animais exibem o nível de bem-estar através de reações comportamentais e fisiológicas como resposta a manejos realizados por humanos e essas reações podem ser mensuradas e avaliadas. Com essas reações, é impossível considerar a importância do conceito de bem-estar e suas implicações complexas na interação homemanimal.

\section{Conceitos de bem-estar}

O bem-estar animal é uma temática complexa, com dimensões científicas, éticas, econômicas, culturais e políticas (OIE, 2014). Em sua essência, o conceito de bem-estar animal resulta da aplicação de práticas de produção animal aceitáveis do ponto de vista ético. Uma definição objetiva de bem-estar é necessária para a utilização científica e profissional do conceito (Broom and Molento, 2004) e para podermos delimitar o que é bom para os animais, pois existem várias espécies com comportamentos, preferências e necessidades distintas uma das outras e de nós humanos (Pinheiro and Brito, $\underline{2009)}$.

O bem-estar deve ser definido de forma que permita pronta relação com outros conceitos, tais como: necessidades, liberdades, felicidade, adaptação, controle, capacidade de previsão, sentimentos, sofrimento, dor, ansiedade, medo, tédio, estresse e saúde (Broom and Molento, 2004).

Organização Mundial de Saúde Animal (OIE, 2014) determina que o termo "bem-estar animal" denota como um animal lida com as condições de seu ambiente. Um animal está em bom estado de bem-estar se, a partir de evidências científicas, seja constatado que está saudável, confortável, bem nutrido, seguro, capaz de expressar comportamento natural e que não sofre sensações desagradáveis de dor, medo e estresse. Outro conceito definido por Broom (1986) determina que o bem-estar de um indivíduo é o estado em relação às suas tentativas de adaptar-se ao seu ambiente. Buscando a praticidade para a avaliação de sistemas produtivos, podemos aplicar este conceito entendendo o bem-estar como o grau de dificuldade que um animal enfrenta (e demonstra) para viver onde está (BRASIL, 2016). Esta definição sugere que bem-estar é uma qualidade inerente aos animais, não uma condição dada pelo homem a estes (Broom, 1991a). Também descreve uma característica do indivíduo em um dado momento, localizando o estado do animal dentro de uma escala de muito bom a muito ruim e pode ser avaliado cientificamente a partir do estado biológico do animal e de suas preferências (Broom, 1991a, Broom and Molento, 2004).

As respostas adaptativas ajudam o animal a controlar suas interações com seu ambiente e a manter a estabilidade mental e corporal (Broom, 1991b). Segundo (Pinheiro and Brito, 2009) podem existir três situações diferentes em relação ao processo de adaptação ao ambiente: a adaptação ao meio ambiente é impossível (o animal vai morrer ou ficar doente, e como resultado, a mortalidade e a incidência de doenças e lesões causadas pelo ambiente são indicadores de falta de bem-estar); a adaptação ao ambiente pode ser possível, mas representa um custo biológico alto para o animal (estresse) ou um animal pode estar em um ambiente em que a adaptação não é apenas possível, mas também é fácil para o animal (o bem-estar dos animais pode ser considerado satisfatório).

Uma forma prática de avaliar o bem-estar de animais de produção é a partir do conceito das "cinco liberdades", elaborado pela "Farm Animal Welfare Council" (FAWC, 2009). As "cinco liberdades" formam uma base lógica para a avaliação do bem-estar dentro de qualquer sistema e direcionam as ações necessárias para manter o bem-estar dentro dos limites em uma produção 
pecuária eficiente (Code of Recommendations for the Welfare of Livestock: Sheep, 2013). As cinco liberdades exploram a existência de indicadores que abrangem todos os aspectos de bem-estar animal (Llonch et al., 2015) e são as diretrizes que devem reger o bem-estar dos animais (OIE, 2014). De acordo com estas liberdades os criadores/tratadores que têm animais sob seus cuidados devem: planejar de forma cuidadosa e responsável; possuir conhecimentos e prática comprovada no manejo de animais; assegurar que a "estrutura e equipamento" das instalações sejam adequadas para garantir a segurança e o bem-estar dos animais; manejar e transportar os animais de forma apropriada e abater os animais sem sofrimento. O conceito das cinco liberdades estava implícito no relatório de Brambel. Posteriormente a ideia foi refinada e publicada pela "Farm Animal Welfare Council" no final de 1970 de uma forma muito semelhante à versão atual. As Cinco Liberdades têm alcançado a população mundial e podem ser encontradas, as vezes com pequenas modificações, em legislações nacionais e como garantia e marketing de propriedades (FAWC, 2009).

Brambell (1965) elaborou um relatório fornecendo diretrizes para o bem-estar de animais mantidos em sistemas de criação intensivos. Esse relatório foi resultado da solicitação do Ministro da Agricultura, Pescas e Alimentos e do Secretário de Estado da Escócia "Para examinar as condições em que os animais eram mantidos em sistemas de criação intensivos, avaliar se deveriam ser definidas diretrizes visando o bem-estar e, caso fossem necessárias, determinar quais deveriam ser as diretrizes". Uma comissão foi nomeada para visitar propriedades de diversas espécies animais com sistemas de criação intensivos e, posteriormente discutir e definir quais deveriam ser a diretrizes que, ao mesmo tempo em que deveriam permitir o desenvolvimento progressivo de sistemas intensivos, também deveriam proporcionar bom grau de bem-estar aos animais.

O relatório Brambell promoveu várias mudanças, entre elas: legislação robusta para proteger os animais de produção; códigos de recomendações para o bem-estar dos animais; avanços significativos na ciência do bem-estar animal; maior ênfase no bem-estar nos currículos de estudantes de ciências agrárias e em programas de formação para os produtores e tratadores; maior vigilância do bem-estar por órgãos competentes; melhorias gerais na pecuária e em manejos e aumento da consciência e das expectativas de alguns consumidores sobre bem-estar dos animais de produção (FAWC, 2009).

As "Cinco Liberdades" são princípios cujos ideais utópicos podem ser utilizados como diretrizes para avaliação das práticas de manejo (BRASIL, 2016) e informam as disposições que devem ser realizadas pelos tratadores com o objetivo de evitar sofrimento desnecessário e promover o bem estar adequado (FAWC, 2009). De acordo com o conceito das "Cinco Liberdades", os animais devem estar (FAWC, 2009):

1. Livres de fome e sede - pelo fácil acesso à água e a uma dieta para manter a saúde e o vigor;

2. Livres de desconforto - proporcionando um ambiente adequado;

3. Livres de dor, ferimentos e doenças - por prevenção ou rápido diagnóstico e tratamento;

4. Livres para expressar comportamentos normais - proporcionando espaço suficiente, instalações adequadas e a companhia de animais da mesma espécie;

5. Livres de medo e de angústia - garantindo condições e tratamentos que evitem sofrimento mental.

\section{Ética e moral}

A tendência da população mundial em demandar dos governos padrões mínimos de bemestar animal nas cadeias produtivas ocorre porque as questões envolvidas possuem forte presença nos códigos morais e éticos de vários países, sendo que o tratamento apropriado dos animais não é mais aceito como alternativa de livre escolha (BRASIL, 2016).

Segundo Pinheiro and Brito (2009) o ser humano forma julgamentos éticos e morais usando o conhecimento acumulado, não sendo a ética natural, ou seja, a criança não nasce com seus conceitos formados de como viver em sociedade, mas é aprendido. Portanto, ética e moral, pela própria etimologia, diz respeito a uma realidade humana que é construída histórica e socialmente a partir das relações coletivas dos seres humanos nas sociedades onde nascem e vivem.

A avaliação do bem-estar sobre determinado aspecto deve ser objetiva e completamente separada de considerações éticas, mas após tais avaliações podem ser tomadas decisões éticas (Broom, 1991b). Por outro lado, todo o conceito de bem-estar animal e sua avaliação podem envolver valores e julgamentos e podem ser 
tomadas decisões éticas sobre como os animais devem ser tratados (Carenzi and Verga, 2009). Posto isso, para discutir as preocupações éticas sobre o tratamento de animais, os cientistas precisam de reflexões éticas para complementar suas informações empíricas e os filósofos precisam basear suas argumentações em conhecimentos sólidos sobre os animais e nas práticas com animais (Carenzi and Verga, 2009).

\section{Estresse}

Nos sistemas modernos de produção os animais são confrontados com situações desafiadoras, provocando estresse comportamental ou fisiológico (Ligout et al., 2011). A redução do estresse durante o manejo melhora a produtividade e evita alterações fisiológicas que poderiam confundir os resultados de pesquisa ou diminuir a produtividade (Grandin, 2014). Além disso, modificações em tipo e quantidade de manejo, bem como da idade em que ocorrem, podem facilitar o manejo e melhorar o bem-estar animal (Goddard et al., 2000). Em contrapartida, a interpretação para as respostas de estresse agudo em termos de bem-estar é difícil, pois geralmente é uma resposta adaptativa normal (Fell et al., 1986). Pesquisadores de etologia aplicada tentam desenvolver procedimentos efetivos para melhorar as reações de animais em relação a humanos que sejam eficientes e duráveis (Krohn et al., 2001), haja vista que os animais podem perceber diferentemente as práticas de manejo conforme suas experiências anteriores e suscetibilidade ao estresse (Gómez et al., 2010). A organização da resposta ao agente estressor não é apenas função da percepção do referido agente, mas também de experiências passadas, o que permite o reconhecimento de uma dimensão psicológica do estresse (Gómez et al., 2010).

Broom and Molento (2004) determinaram que a palavra estresse devesse ser utilizada para descrever aquela porção do bem-estar pobre que se refere à falência nas tentativas de enfrentar as dificuldades, então sempre que existe estresse o bem-estar torna-se pobre. Estresse refere-se somente a situações nas quais existe falência de adaptação, porém bem-estar pobre se refere ao estado de um animal, seja em condições onde existe falência de adaptação ou quando o indivíduo está encontrando dificuldades em se adaptar.

Segundo Pinheiro and Brito (2009) em resposta ao estresse cada animal pode utilizar as defesas biológicas disponíveis de formas bem diferentes, não havendo resposta específica aplicada a cada estímulo estressante, o que torna muito difícil mensurar clinicamente o estresse. Uma opção para tentar mensurá-lo é a partir da etologia, que é uma ciência que tem por função analisar as leis que regem as manifestações vitais dos animais em condições naturais e artificiais, bem como analisar suas causas. Quando o BEA é afetado, seja por alterações no meio ambiente, presença de lesão, doenças, tensão ou outras, ocorrem alterações da fisiologia e das condições físicas e psicológicas do animal, o que, por sua vez, resulta em modificações do comportamento. Desta forma, devem-se conhecer bem os parâmetros comportamentais normais para as espécies e raças que serão avaliadas, em determinadas condições ambientais.

\section{Legislação e recomendações de bem-estar de ovinos}

A Organização Mundial de Saúde Animal (World Organisation for Animal Health (OIE, 2014) é a única organização intergovernamental mundial responsável pela criação de diretrizes intergovernamentais na área de bem-estar animal.

A percepção de bem-estar animal e o que constitui ou não constitui um ato de crueldade contra os animais difere de região e de cultura para outra (OIE, 2014). Com o auxílio de inúmeros especialistas, a OIE produziu normas de bem-estar baseadas em pesquisas e esses resultados levaram ao consenso entre todos os países membros da OIE, apoiando a adoção de tais diretrizes. Com essas normas foram elaborados vários capítulos abordando diferentes sistemas de produção, entre eles: bovinos e aves de corte, bovinos leiteiros, equinos usados para trabalho e peixes. Atualmente tem avançado a elaboração de diretrizes relativas às galinhas poedeiras e aos suínos (OIE, 2014), ou seja, não existem normas específicas para a produção de ovinos na Organização Mundial de Saúde Animal, devendo ser seguidas as recomendações para bem-estar de forma geral.

$\mathrm{Na}$ Europa foram elaboradas recomendações referentes à legislação de bem-estar de ovinos com $o$ intuito de fornecer uma ferramenta de apoio mais clara, explícita e que possa ser compreendida por todos os envolvidos com pecuária. Uma parte das orientações descritas são recomendações e devem ser entendidas como tal, embora estas em conjunto com a legislação em vigor sejam de fato um "Manual" de Bem-Estar Animal. As 
recomendações estão disponíveis em CAP (2006) e Code of Recommendations for the Welfare of Livestock: Sheep (2013).

No Brasil, o Ministério da Agricultura, Pecuária e Abastecimento (MAPA) conta com uma Comissão Técnica de Bem-estar Animal que desenvolve ações visando o estabelecimento de normas que favoreçam o bem-estar animal e, para isso, tem demandado de instituições de pesquisa a coordenação de projetos específicos nesta área, que forneçam embasamento científico aos documentos normativos e protocolos, conforme as diretrizes oficiais (Pinheiro and Brito, 2009). As autoridades oficiais do Brasil, em conjunto com universidades, centros de pesquisa e setores privados têm realizado importante progresso em bem-estar animal e também têm sido conduzidos programas de treinamento e divulgação de boas práticas na criação animal (Huertas et al., 2014).

O MAPA, em conjunto com outras instituições, está desenvolvendo o "Projeto de bem-estar na ovinocultura". Este projeto, desenhado com a EMBRAPA pecuária sul (e com apoio da Associação Brasileira de Criadores de Ovinos, Universidade de São Paulo, Universidade Positivo e Universidade Federal de Pelotas) tem o objetivo de validar o protocolo de avaliação de bem-estar de ovinos para a realidade nacional, capacitação dos técnicos de campo em sua aplicação, bem como fomentar a pesquisa e o desenvolvimento de materiais técnicoinformativos sobre o manejo da espécie. Sua finalização está prevista para 2020.

\section{Bem-estar no Brasil}

A legislação de bem-estar animal no Brasil teve inicio com o Decreto ${ }^{\circ} 24.645$ de julho de 1934, que estabelece medidas de proteção animal. Nossa atual Constituição Federal de 1988, no seu $\operatorname{artigo~}^{\circ} 225$, dota o poder público de competência para proteger a fauna e a flora, vedando práticas que submetam os animais a crueldade.

Em 2008 foi criada pelo MAPA a Comissão Técnica Permanente de Bem-Estar Animal CTBEA (criada pela Portaria $n^{\circ} 185$ de 2008 e atualizada pela portaria $\mathrm{n}^{\circ} 524$ de 2011). Dentre as atribuições da CTBEA estão à divulgação e a proposição de boas práticas de manejo, o alinhamento da legislação brasileira com os avanços científicos e os critérios estabelecidos pelos acordos internacionais dos quais o Brasil é signatário, bem como preparar e estimular o setor agropecuário brasileiro para o atendimento às novas exigências da sociedade brasileira e consumidores dos mercados importadores.

O MAPA estabelece que os conceitos mais aceitos no meio científico são o de Broom (1986) e o Conceito das "Cinco Liberdades" da FAWC (2009). O conceito de orientação para as ações da Comissão Técnica Permanente de Bem-Estar Animal é o conceito de bem-estar utilizado pela Organização Mundial de Saúde Animal onde um bom grau de bem-estar animal significa um animal que está seguro, saudável, confortável, bem nutrido, livre para expressar comportamentos naturais e sem sofrer de estados mentais negativos, como dor, frustração e estresse.

O Brasil, como membro da Organização Mundial de Saúde Animal, organismo internacional de referência para o bem-estar animal, atende as diretrizes estabelecidas no Código Sanitário para Animais Terrestres. Além de atender tais diretrizes, o MAPA regulamenta o bem-estar animal através do decreto e das instruções normativas (BRASIL, 2016):

- Decreto no 24.645 de 1934 - Estabelece Medidas de Proteção Animal.

- Decreto n 30.691 de 1952 - Regulamento da Inspeção Industrial e Sanitária de Produtos de Origem Animal: torna o estabelecimento industrial responsável pela garantia do bem-estar dos animais, da chegada dos animais na indústria até o abate, além de prever sanções a estes quando do não cumprimento deste e outros requisitos.

- Lei ${ }^{\circ} 9.605$, de 12 de fevereiro de 1998 - Lei de crimes ambientais.

- Instrução Normativa $\mathrm{n}^{\circ} 03$ de 2000 Regulamento Técnico de Métodos de Insensibilização para o Abate Humanitário de Animais de Açougue: regulamenta os procedimentos de manejo pré-abate e abate humanitário, desde a chegada dos animais no estabelecimento industrial até $\mathrm{o}$ abate.

- Instrução Normativa $n^{\circ} 56$ de 2008 Estabelece os procedimentos gerais de Recomendações de Boas Práticas de Bem-Estar para Animais de Produção e de Interesse Econômico, abrangendo os sistemas de produção e o transporte.

- Lei $N^{\circ} 11.794$, de 8 de outubro de 2008 Estabelece procedimentos para o uso científico de animais. 
- Instrução Normativa ${ }^{\circ} 10$ de 2010 - Aprova Regulamento Técnico para exportação de ruminantes vivos para o abate.

\section{Como medir o bem-estar animal?}

O desafio para a pesquisa em bem-estar é descobrir como os animais "sentem", o quanto isso importa para eles, quais são as necessidades específicas dos animais e como essas necessidades podem ser satisfeitas pelo ambiente em que vivem (Carenzi and Verga, 2009).

Broom and Molento (2004) explicam que quando um animal se encontra em desajuste homeostático real ou potencial, ou quando tem de executar uma ação devido a alguma situação ambiental, diz-se que este animal tem uma necessidade. Quando as necessidades são satisfeitas, o bem-estar é mais adequado e o animal experimenta melhores sentimentos que em situações nas quais as necessidades não são satisfeitas. Informações a respeito dos sentimentos podem ser obtidas através de estudos de preferência, mas devem ser complementadas com as outras informações de bem-estar.

As necessidades podem ser divididas em diferentes categorias: as necessidades ambientais, como alojamento e manejo (que incluem reprodução, higiene, transporte e enriquecimento ambiental) e as necessidades fisiológicas e comportamentais, que incluem a possibilidade de expressar as funções biológicas essenciais e o repertório comportamental, que também depende da interação com os seres humanos e da seleção genética de indivíduos criados para características desejáveis (Carenzi and Verga, 2009).

A avaliação comportamental é bastante importante para medir o bem-estar, mas deve ser combinada com variáveis fisiológicas, imunológicas, lesões, doenças, riscos de morte, crescimento e reprodução a fim de obter informação completa (Broom, 1991b) e para saber quão bom ou ruim está o bem-estar dos animais (Broom, 1991a). Como afirmam Pinheiro and Brito (2009) um animal está em bom estado de bem-estar se ele está saudável, confortável, bem alimentado, seguro, hábil para expressar seu comportamento normal, e não estiver sofrendo estados desagradáveis de dor, medo ou aflição. Sendo assim, o bem-estar animal requer: prevenção de doença, tratamento veterinário, manejo nutricional, instalações adequadas e finalmente um abate ou eutanásia humanitário.
Barnett and Hemsworth (1990) afirmaram que as avaliações de bem-estar estão baseadas em mudanças fisiológicas ou comportamentais. Contudo, a mudança em si não é um indicador de mudança no bem-estar, pois os comportamentos e a fisiologia são ajustados continuamente para manter a homeostase após variações ambientais e os animais não mudam continuamente o bem-estar como resposta a estes ajustes. Então, é importante questionar "em qual nível de mudança (na fisiologia ou no comportamento) o bem-estar está em risco?".

Dentre 18 possíveis indicadores de bem-estar disponíveis na literatura, nove deles seriam teoricamente possíveis de avaliar no abatedouro, são eles: limpeza corporal, lesão na carcaça, diarreia, lesão ou irritação na pele, castração, marcação na orelha, corte da cauda e animais registrados como "obviamente doente" (Llonch et al., 2015). Broom and Molento (2004) afirmaram que, independentemente do tipo de medição (Tabela 1), os dados coletados em estudos de bemestar animal fornecem informações sobre a posição do animal em uma escala de bem-estar, variando de um grau muito alto até muito baixo. Entretanto, em qualquer avaliação de bem-estar devem ser consideradas as variações individuais ao se enfrentar adversidades e os efeitos que as adversidades exercem sobre os animais.

Tabela 1. Parâmetros para mensuração de bem-estar (Broom and Molento, 2004).

Demonstração de uma variedade de comportamentos normais

Grau em que comportamentos fortemente preferidos podem ser apresentados

Indicadores fisiológicos de prazer

Indicadores comportamentais de prazer

Expectativa de vida reduzida

Crescimento ou reprodução reduzidos

Danos corporais

Doença

Imunossupressão

Tentativas fisiológicas de adaptação

Tentativas comportamentais de adaptação

Doenças comportamentais

Auto-narcotização

Grau de aversão comportamental

Grau de supressão de comportamento normal

Grau de prevenção de processos fisiológicos normais e de desenvolvimento anatômico

Os efeitos sobre o bem-estar incluem aqueles provenientes de doença, traumatismos, fome, 
estimulação benéfica, interações sociais, condições de alojamento, tratamento inadequado, manejo, transporte, procedimentos laboratoriais, mutilações variadas, tratamento veterinário ou alterações genéticas através de seleção genética convencional ou por engenharia genética (Broom and Molento, 2004).

\section{Comportamentos de ovinos}

Muitos problemas atuais de criação de animais não podem ser solucionados investigando a nutrição, fisiologia ou controlando doenças, mas exigem investigações comportamentais para posteriores tomadas de decisões (Broom and Molento, 2004). Seu conhecimento pode servir como guia para adequar o ambiente e obter ótimas condições de criação, alimentação e máxima eficiência da produção (Barros et al., 2011).

O termo etologia significa a observação e descrição detalhada do comportamento com o objetivo de descobrir como os mecanismos biológicos funcionam e pode ser um indicador de bem-estar bom ou ruim em qualquer animal (Broom and Molento, 2004).

Caroprese (2008) citando vários autores afirmou que os ovinos são animais gregários, não agressivos, que experimentam medo, ansiedade e frustração durante manejos simples e que não se adaptam bem a confinamentos. Por serem altamente sociais, o isolamento do rebanho é uma das situações mais estressantes na prática pecuária moderna e a avaliação do comportamento e da frequência cardíaca fornecem uma boa indicação das reações dos animais(Baldock and Sibly, 1990).

Os animais podem, em algum momento da vida, apresentar comportamentos anormais, que são atividades que mostram funcionamento anormal ou prejudicado na ação ou no comportamento. Comportamentos anormais podem indicar patologias, facilitando o diagnóstico e tratamento, mas a maioria dos diagnósticos comportamentais para animais de produção são descritivos e não específicos (Broom and Molento, 2004).

\section{Estereotipias}

Um estereótipo é uma sequência repetida, relativamente invariante de movimentos que não tem nenhum propósito óbvio (Broom and Molento, 2004). Enquanto a causa das estereotipias não é clara, a função das mesmas é menos clara ainda (Barnett and Hemsworth, 1990). Estereótipos são mostrados em situações em que o indivíduo não tem controle sobre o ambiente, especialmente naqueles que são obviamente frustrantes, ameaçadores ou onde há severa falta de estimulação (Broom, 1991a). Animais com privações severas ou submetidos a frustração extrema demonstram estereotipias, que pode ser interpretada como uma indicação de problema psicológico temporário ou de longa duração (Broom, 1991b). De maneira similar à resposta fisiológica ao estresse, o desenvolvimento de estereotipias pode estar relacionado a mecanismos que ajudam os animais a lidar com mudanças ambientais (Wiepkema et al., 1984). Se isso estiver correto, os animais em situações semelhantes que não desenvolvem estereotipias estão tendo dificuldade de adaptação ou utilizam mecanismos comportamentais diferentes como resposta às mudanças ambientais (Barnett and Hemsworth, 1990)? Independente da causa, estereotipias são mostradas em situações difíceis para o animal, indicando que o bem-estar está pobre e uma grande quantidade de estereotipias indica bem-estar mais pobre do que uma estereotipia ocasional (Broom, 1991a).

\section{Instalações para ovinos}

O Bem-Estar Animal é indiscutivelmente dependente de um bom manejo e de um correto planejamento da exploração. Nesse caso, as instalações modernas e as produções intensivas têm ganhado a atenção de cientistas, com foco no microclima no interior das instalações, nas práticas de manejo e no bem-estar dos animais (Caroprese, 2008). Pequenas alterações de manejo e instalações, mesmo associadas a baixos investimentos, podem representar uma elevação importante no padrão de bem-estar dos animais, minimizando perdas nos sistemas produtivos.

Segundo Broom (1991a) para desenvolver bons sistemas de manejo e bons alojamentos é muito importante desenvolver estudos sobre as preferências dos animais, pois é necessário descobrir as preferências para proporcinar bons cuidados. Esses estudos de preferência são complementares às outras medidas de bem-estar citadas anteriormente.

Caroprese (2008) afirma que, como os ovinos não se adaptam bem a confinamentos, sua saúde e bem-estar podem ser melhorados, nas instalações, com a possibilidade de acesso a áreas externas; proteção contra temperaturas extremas e controle 
do clima; espaço suficiente; higiene, regime de luz e ventilação adequadas. Por outro lado, a radiação solar direta aumenta a frequência respiratória, temperatura retal, quantidade de comportamentos inativos, mobilização de reservas corporais e diminui a proliferação de linfócitos e a capacidade de defesa mamária. $\mathrm{O}$ estresse térmico pode ser amenizado com o fornecimento de ventilação adequada, que vai diminuir a poluição do ar (amônia e dióxido de carbono proveniente da respiração e da decomposição das fezes) e melhorar as trocas térmicas entre os animais e o ambiente (Caroprese, 2008). Pinheiro and Brito (2009) descreveram que as instalações destinadas a alojar os animais ou os currais de manejo devem ser simples, eficientes, de baixo custo e devem proporcionar aos animais condições de conforto, espaço e proteção de um ambiente limpo, seco e de boas condições sanitárias para evitar doenças. Também devem ser construídas levando em consideração os comportamentos da espécie que será criada, levando a um trabalho mais fácil, seguro, eficiente e menos estressante para os animais e para as pessoas envolvidas.

O confinamento ou semi confinamento pode não ocasionar diferenças endócrinas ou imunológicas em ovelhas, mas ovelhas semi confinadas se deslocam mais e apresentaram menor contagem de células somáticas e melhor qualidade do leite (Casamassima et al., 2001). A redução severa de espaço por animal nos confinamentos pode levar à diminuição da possibilidade de se exercitar, levando ao bemestar pobre (Broom and Molento, 2004). Também leva a uma pior higiene, pois prejudica a qualidade do ar devido ao aumento da quantidade de microrganismos, gases (amônia e dióxido de carbono) e poeira (Caroprese, 2008). Fezes e lixo são responsáveis pela produção de $57-76 \%$ de partículas de poeira nas instalações, podendo ser considerada o principal transporte de microrganismos no ar (Hartung, 1994). O uso de bentonita para tratar o lixo $\left(0.5 \mathrm{~kg} / \mathrm{m}^{2}\right.$ de lixo) pode dificultar a sobrevivência e multiplicação de microorganismos na cama dos animais (Sevi et al., 2001). A (CAP, 2006) determinou as dimensões mínimas necessárias para os animais de diferentes categorias (Tabela 2). Pensando em ovinos com 45-60 kg, um espaço de $0.8 \mathrm{~m}^{2} /$ animal é necessário em baias totalmente ripadas, devendo aumentar $30 \%$ por animal em baias com cama coberta por palha e $15 \%$ para animais tosquiados (Loynes, 1983).

Tabela 2. Necessidades mínimas de espaço para os animais em diferentes categorias (CAP, 2006).

Fêmeas $(60-90 \mathrm{Kg})$

Fêmeas depois do parto com cordeiros até seis semanas de idade

Fêmeas em área montanhosa $(45-65 \mathrm{~kg})$

Fêmeas em área montanhosa depois do parto com cordeiros até seis semanas de idade

Cordeiros até 12 semanas de idade

Animais de 12 semanas a 12 meses de idade

Carneiros não castrados
1,2-1,4 $\mathrm{m}^{2}$ de espaço por fêmea durante a prenhez

2,0-2,2 $\mathrm{m}^{2}$ de espaço por fêmea e cordeiro

1,0-1,2 $\mathrm{m}^{2}$ de espaço por fêmea durante a prenhez

1,8-2,0 $\mathrm{m}^{2}$ de espaço por fêmea e cordeiro

0,5-1,6 $\mathrm{m}^{2}$ de espaço por cordeiro

$0,75-0,9 \mathrm{~m}^{2}$ de espaço por animal

$1,5-2,0 \mathrm{~m}^{2}$

\section{Conclusão}

O bem-estar de ovinos tem sido bastante pesquisado ao redor do mundo e, como consequência, tem sido produzido conhecimento capaz de fornecer subsídio para a elaboração de diretrizes para os diversos tipos de produção de ovinos. No Brasil ainda não existe legislação específica para ovinos e a quantidade de pesquisas é insuficiente para determinar as necessidades das diferentes raças, nos diversos sistemas de produção e condições climáticas brasileiras, dificultando a implementação de sistemas adequados para os animais.

\section{Referências Bibliográficas}

Baldock, N. M. \& Sibly, R. M. 1990. Effects of handling and transportation on the heart rate and behaviour of sheep. Applied Animal Behaviour Science, 28, 15-39.

Barnett, J. L. \& Hemsworth, P. H. 1990. The validity of physiological and behavioural measures of animal welfare. Applied Animal Behaviour Science, 25, 177-187. 
Barros, C. S., Monteiro, A. L. G., Dittrich, J. R., Fernandes, M. A. M. \& Pinto, S. 2011. Comportamento social de ovinos: apresentação de técnicas. Scientia Agraria Paranaenis, 10, 5-20.

Boivin, X., Nowak, R. \& Garcia, A. T. 2001. The presence of the dam affects the efficiency of gentling and feeding on the early establishment of the stockperson-lamb relationship. Applied Animal Behaviour Science, 72, 89-103.

Brambell, F. W. R. 1965. Report of the Technical Committee to Enquire into the Welfare of Animals kept under Intensive Husbandry Systems. HM Stationery Office, London.

Broom, D. M. 1986. Indicators of poor welfare. British Veterinary Journal, 142, 524-526.

Broom, D. M. 1991a. Animal welfare: concepts and measurement. Journal of Animal Science, 69, 4167-4175.

Broom, D. M. 1991b. Assessing welfare and suffering. Behavioural Processes, 25, 117-123.

Broom, D. M. \& Molento, C. F. M. 2004. Animal welfare: concept and related issues-review. Archives of Veterinary Science, 9, 1-11.

Carenzi, C. \& Verga, M. 2009. Animal welfare: review of the scientific concept and definition. Italian Journal of Animal Science, 8, 21-30.

Caroprese, M. 2008. Sheep housing and welfare. Small Ruminant Research, 76, 21-25.

Casamassima, D., Sevi, A., Palazzo, M., Ramacciato, R., Colella, G. E. \& Bellitti, A. 2001. Effects of two different housing systems on behavior, physiology and milk yield of Comisana ewes. Small Ruminant Research, 41, 151-161.

Euclides Filho, K. 2004. Supply chain approach to sustainable beef production from a Brazilian perspective. Livestock Production Science, 90, 53-61.

FAWC. 2009. Farm animal welfare in Great Britain: Past, present and future. Farm Animal Welfare Council, England.

Fell, L. R., Wells, R. \& Shutt, D. A. 1986. Stress in calves castrated surgically or by the application of rubber rings. Australian Veterinary Journal, 63, 16-18.

Goddard, P. J., Fawcett, A. R., Macdonald, A. J. \& Reid, H. W. 2000. The behavioural, physiological and immunological responses of lambs from two rearing systems and two genotypes to exposure to humans. Applied Animal Behaviour Science, 66, 305-321.
Gómez, J. M. D., Fischer, V., Poli, C. H. E. C., Carvalho, P. C. d. F., Pegoraro, E. J. \& Macari, S. 2010. Efeitos da oferta de forragem, do método de pastejo, dos dias de avaliação e da raça no comportamento e temperamento de ovinos. Revista Brasileira de Zootecnia, 39, 1840-1848.

Grandin, T. 2014. Animal welfare and society concerns finding the missing link. Meat Science, 98, 461-469.

Hartung, J. 1994. Environment and animal health. Livestock Housing, Wallingford.

Huertas, S. M., Gallo, C. \& Galindo, F. 2014. Drivers of animal welfare policy in the Americas. Revue Scientifique et Technique, 33, 67-76.

Krohn, C. C., Jago, J. G. \& Boivin, X. 2001. The effect of early handling on the socialisation of young calves to humans. Applied Animal Behaviour Science, 74, 121-133.

Lawrence, A. B. \& Appleby, M. C. 1996. Welfare of extensively farmed animals: principles and practice. Applied Animal Behaviour Science, $49,1-8$.

Ligout, S., Foulquié, D., Sèbe, F., Bouix, J. \& Boissy, A. 2011. Assessment of sociability in farm animals: The use of arena test in lambs. Applied Animal Behaviour Science, 135, 5762.

Llonch, P., King, E. M., Clarke, K. A., Downes, J. M. \& Green, L. E. 2015. A systematic review of animal based indicators of sheep welfare on farm, at market and during transport, and qualitative appraisal of their validity and feasibility for use in UK abattoirs. The Veterinary Journal, 206, 289-297.

Millman, S. T., Duncan, I. J. H., Stauffacher, M. \& Stookey, J. M. 2004. The impact of applied ethologists and the International Society for Applied Ethology in improving animal welfare. Applied Animal Behaviour Science, 86, 299-311.

OIE. 2014. World Organization for Animal Health. World Health Organization.

Pinheiro, A. A. \& Brito, F. I. 2009. Bem-estar e produção animal. EMBRAPA, Sobral.

Probst, J. K., Spengler Neff, A., Leiber, F., Kreuzer, M. \& Hillmann, E. 2012. Gentle touching in early life reduces avoidance distance and slaughter stress in beef cattle. Applied Animal Behaviour Science, 139, 4249. 
Rault, J.-L., Boissy, A. \& Boivin, X. 2011. Separation distress in artificially-reared lambs depends on human presence and the number of conspecifics. Applied Animal Behaviour Science, 132, 42-50.

Romeyer, A. \& Bouissou, M.-F. 1992. Assessment of fear reactions in domestic sheep, and influence of breed and rearing conditions. Applied Animal Behaviour Science, 34, 93-119.

Sevi, A., Taibi, L., Muscio, A., Albenzio, M., Dantone, D. \& Dell Aquila, S. 2001. Quality of ewe milk as affected by stocking density and litter treatment with bentonite. Italian Journal of Food Science, 13, 77-86.

Simplício, A. A., Wander, A. E., Leite, E. R. \& Lopes, E. A. 2004. A caprino-ovinocultura de corte como alternativa para a geração de emprego e renda. In: EMBRAPA (ed.) Embrapa Caprinos. Documentos. EMBRAPA, Mossoró.

Waterhouse, A. 1996. Animal welfare and sustainability of production under extensive conditions - a European perspective. Applied Animal Behaviour Science, 49, 29-40.

Wiepkema, P. R., Cronin, G. M. \& Van Ree, J. M. 1984. Stereotypies and endorphins: functional significance of developing stereotypies in tethered sows. Proceedings of the International Congress on Applied Ethology in Farm Animals.

Wolf, B. T., McBride, S. D., Lewis, R. M., Davies, M. H. \& Haresign, W. 2008. Estimates of the genetic parameters and repeatability of behavioural traits of sheep in an arena test. Applied Animal Behaviour Science, 112, 6880.

\section{Article History:}

Received 17 October 2016

Accepted 11 November 2016

Available on line 1 December 2016

License information: This is an open-access article distributed under the terms of the Creative Commons Attribution License 4.0, which permits unrestricted use, distribution, and reproduction in any medium, provided the original work is properly cited. 